\title{
Diaphragm ultrasonography and pulmonary function tests in patients with spinal cord injury
}

\author{
Fevziye Ünsal Malas ${ }^{1} \cdot$ Füsun Köseoğlu ${ }^{1}$ Murat Kara ${ }^{2} \cdot$ Hilal Ece $^{1} \cdot$ Meltem Aytekin ${ }^{1} \cdot$ Gökhan T. Öztürk $^{1}$ • \\ Levent Özçakar ${ }^{2} \cdot$ Alper M. Ulaşlı $^{1}$
}

Received: 16 November 2018 / Revised: 6 March 2019 / Accepted: 12 March 2019 / Published online: 9 April 2019

(c) International Spinal Cord Society 2019

\begin{abstract}
Study design Cross-sectional study.

Objective To investigate the role of ultrasonographic measurement of the diaphragm thickness on pulmonary function tests in patients with spinal cord injury (SCI).

Setting Rehabilitation center in Ankara, Turkey.

Methods A total of 42 patients (34 M, 8 F) with SCI and 20 able-bodied volunteers $(8 \mathrm{M}, 12 \mathrm{~F}$ ) were enrolled. Patients with SCI were divided into three groups according to their neurological (injury) levels. All participants underwent ultrasonographic measurements for diaphragm thickness on both sides and spirometric tests for pulmonary functions. The thickness ratio of the diaphragm was also calculated.

Results There were seven patients (5 M, 2 F) in C2-C4 injury group, 14 patients (12 M, 2 F) in C5-T5 group, 21 patients $(14 \mathrm{M}, 7 \mathrm{~F})$ in T6-L2 group, and 20 able-bodied volunteers ( $8 \mathrm{M}, 12 \mathrm{~F})$. The diaphragms of C2-C4 group were thicker than those of the controls at end-inspirium on the right side $(2.7 \pm 0.7 \mathrm{~mm}$ vs. $2.0 \pm 0.5 \mathrm{~mm} ; p=0.035)$. The thickness ratios of $\mathrm{C} 2-\mathrm{C} 4$ group were lower than those of controls on the right $(0.8 \pm 0.4$ vs. $1.5 \pm 0.5 ; p=0.005)$ and left $(0.8 \pm 0.5$ vs. $1.6 \pm$ $0.7 ; p=0.003$ ) sides. For all the pulmonary function tests (except for FEV1/FVC); patients with SCI had worse results than controls; and among the SCI groups, the higher the injury level, the worse the results.

Conclusion Although patients with high-level SCI had worse pulmonary function tests and decreased the contractile capacity of the diaphragm, they had thicker diaphragm muscles than controls. This may have been due to the compensatory effect of the diaphragm (performing its maximum contraction capacity and increasing frequency of inspiration).
\end{abstract}

\section{Introduction}

Spinal cord injury (SCI) is a common cause of chronic respiratory failure and the severity of diaphragm paresis is an important determinant of long-term respiratory problems. Pulmonary restriction [1] or obstruction [2], and ventilatory muscle weakness are commonly seen clinical problems in patients with high-cervical spinal injury. Chest wall compliance decreases and-with denervation of abdominal

Fevziye Ünsal Malas

fevunsal@hotmail.com

1 Ankara Physical Medicine and Rehabilitation Training and Research Hospital, Ankara, Turkey

2 Hacettepe University Medical School, Department of Physical and Rehabilitation Medicine, Ankara, Turkey muscles-inspiratory and expiratory lung functions are also affected in association with the level of SCI [3].

The diaphragm is a dome-shaped structure that performs the majority of the work during breathing. It is adjacent to the lower rib cage in a region known as the zone of apposition [4]. Although pulmonary function tests are helpful, they provide an indirect measurement of its function $[4,5]$ and they are also dependent on patient effort-yielding false positive measurements.

Owing to its several advantages (e.g., convenient, repeatable, does not contain radiation exposure) [6, 7], ultrasound (US) imaging has been used to assess respiratory muscles in humans, including the relationship between lung volumes and diaphragmatic thickness [6, 8-10]. Although there are studies about diaphragm US imaging and pulmonary function tests in able-bodied participants $[6,11]$ and in stroke patients $[12,13]$; to our best notice, very few studies [14-16] have investigated the relationship between 
diaphragm muscle and spirometric tests in patients with SCI. One of them is a report of three patients with tetraplegia [14] and the other one comprises evaluation of 10 paralympic rugby players with SCI [16]. Neither of them assessed the aforementioned relationship according to different neurological levels.

Accordingly, in this study, we aimed to explore the role of US measurement of the diaphragm thickness on physiologic respiratory performance measures in a larger group of patients with different levels of SCI.

\section{Methods}

Forty-two patients (34 M, 8 F) with SCI who had been admitted to our rehabilitation center were enrolled in this study. Twenty able-bodied volunteers $(8 \mathrm{M}, 12 \mathrm{~F})$ were also recruited as a control group. Participants underwent a complete medical assessment including medical history, physical and neurological examination, postero-anterior tele-radiograph, resting electrocardiogram and routine laboratory measurements. Exclusion criteria were the previous history of neuromuscular or respiratory diseases, any chronic systemic disorder and cardiopulmonary or abdominal surgery.

Demographic and clinical features of the participants were recorded. Injury severity was classified according to the American Spinal Injury Association Impairment Scale (AIS). We classified the patients with SCI into three groups according to their neurological levels; considering innervation zones of all muscles participating in respiration, especially main inspiratory and expiratory muscles (including intercostals and abdominal muscles) [17]. The first group had $\mathrm{C} 2-\mathrm{C} 4$ injury level-considering phrenic nerve damage. The second group's injury level was C5-T5 level, and the last group had T6-L2 injury level-considering rectus abdominis, external-internal obliques, and transversus abdominis innervation levels. None of the patients had needed respiratory or ventilatory support. The study design was approved by the local Ethics Committee and all patients gave written informed consent to participate in the study. All 62 participants underwent US measurements of both left and right hemi-diaphragms and also spirometric tests for pulmonary function assessment.

\section{Resting spirometry}

Conventional spirometric measurements were performed using a spirometer (Sensormedix Vmax29, Yorba Linda, CA, USA). Tests were performed with the patient in the seated position and repeated at least three trials. Instructions were given and maneuvers were demonstrated for the participants before testing. Acceptable recordings were achieved from each maneuver and best values were used for the analyses. Spirometric parameters included forced vital capacity (FVC), forced expiratory volume in one second (FEV1), the ratio of FEV1 to FVC (FEV1/FVC), forced expiratory flow rate $25-75 \%$ (FEF $25-75 \%$ ), peak expiratory flow rate (PEF), maximum voluntary ventilation (MVV). Measurements were expressed as percentages of the normal predicted values.

\section{Ultrasonographic evaluation}

All measurements were done bilaterally using a linear probe (7-12 MHz Logiq P5, GE Medical Systems, Wisconsin, USA). Diaphragm thickness was assessed while all participants lied supine with their knees extended in a relaxed position. To obtain an intercostal view, the transducer was positioned longitudinally over the anterior axillary line at approximately the 9th intercostal space where there was the least encroachment of the lung. Patients were examined during quiet respiration and sniff maneuver (maximum inspiration). The diaphragm was identified by its characteristic three-layered appearance composed of two hyperechoic lines of pleural and peritoneal fascia and hypoechoic muscle tissue in between. Three diaphragm thickness measurements i.e. at the end of expiration, at the end of inspiration and during maximum inspiration (sniff maneuver) were recorded (Fig. 1). For thickness ratio, the following formula was used;

$\left[\right.$ thickness $_{\max -\text { inspiration }}-$ thickness $\left._{\text {end-expiration }}\right] /$ thickness end-expiration

\section{Statistical analysis}

Statistical analysis was done by using SPSS version 16.0. Data are expressed as mean \pm standard deviation or median (min-max). Shapiro-Wilk's test was used to determine whether demographic and clinical data were normally distributed. Thereafter, those variables were compared with ANOVA (with Bonferroni-corrected post hoc tests), Kruskal-Wallis or Chi-square test among the groups, where appropriate. Right and left side diaphragm measurements (within groups) were compared using paired $t$-test or Wilcoxon test, where appropriate. Statistical significance was set at $p<0.05$.

\section{Results}

Characteristics of the participants are given in Table 1 . There were seven patients ( $5 \mathrm{M}, 2 \mathrm{~F}$ ) in $\mathrm{C} 2-\mathrm{C} 4$ injury group (one C3 ASIA D, five C4 ASIA A and one C4 ASIA B). 


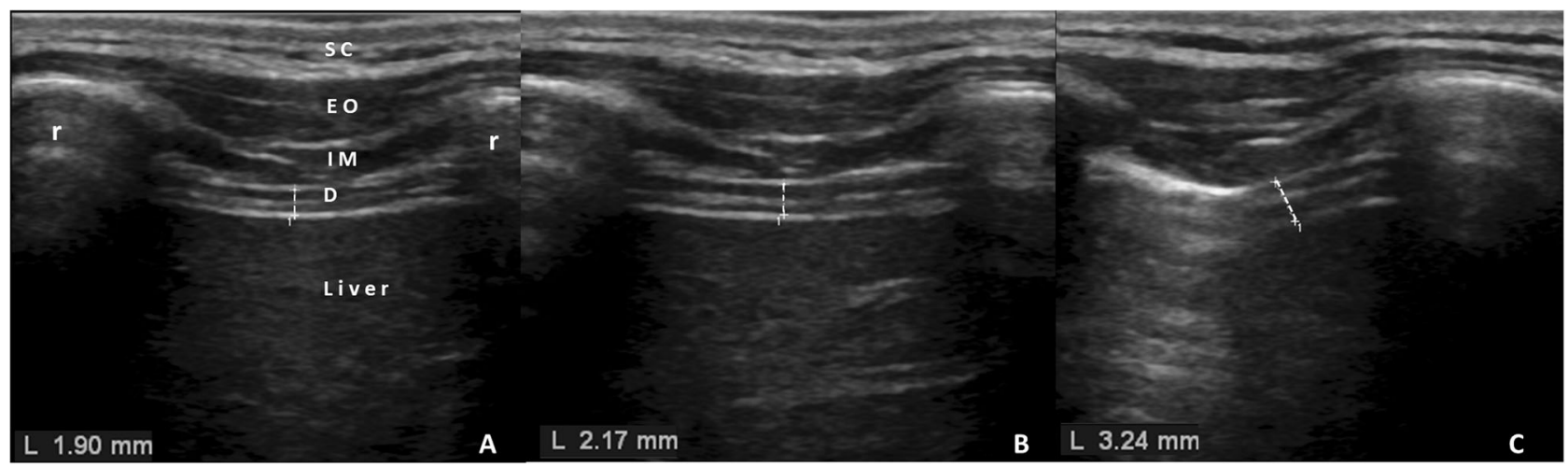

Fig. 1 Ultrasound imaging of the diaphragm over the intercostal space (zone of apposition) for measuring its thickness at end-expiration (a), end-inspiration (b) and during maximum inspiration (c). r: Rib, SC: subcutaneous tissue, EO: abdominal external oblique muscle, IM: intercostal muscle D: diaphragm
Table 1 Characteristics of the participants

\begin{tabular}{llllll}
\hline Characteristic & $\begin{array}{l}\text { Able-bodied } \\
(N=20)\end{array}$ & $\begin{array}{l}\mathrm{C} 2-\mathrm{C} 4 \\
(N=7)\end{array}$ & $\begin{array}{l}\text { C5-T5 } \\
(N=14)\end{array}$ & $\begin{array}{l}\text { T6-L2 } \\
(N=21)\end{array}$ & $P$ \\
\hline Age (years) & $31.6 \pm 5.1$ & $32.4 \pm 8.5$ & $35.3 \pm 10.9$ & $32.0 \pm 11.8$ & 0.694 \\
BMI (kg/m $\left.{ }^{2}\right)$ & $24.7 \pm 3.6$ & $21.7 \pm 3.1$ & $24.7 \pm 3.9$ & $22.3 \pm 4.9$ & 0.139 \\
Duration (months) & - & $22(2-48)$ & $16(4-120)$ & $21(3-180)$ & 0.480 \\
Gender (M/F) & $8 / 12$ & $5 / 2$ & $12 / 2$ & $17 / 4$ & $\mathbf{0 . 0 1 3}$ \\
Smoking & $6 / 14$ & $2 / 5$ & $4 / 10$ & $14 / 7$ & $\mathbf{0 . 0 2 2}$ \\
Completeness of & - & $5 / 2$ & $9 / 5$ & $9 / 12$ & 0.287 \\
injury & & & & & \\
\hline
\end{tabular}

Values are given as mean $\pm \mathrm{SD}$, median (min-max) or ratio

$A I S$, American Spinal Injury Association Impairment scale, $B M I$ body mass index, $M$ male, $F$ female

${ }^{a}$ The ratio of participants with complete (AIS A)/incomplete (AIS B,C,D) injury

Bold $P$-values indicate statistical significance
There were 14 patients (12 M, $2 \mathrm{~F}$ ) in C5-T5 injury group, 21 patients (14 M, $7 \mathrm{~F}$ ) in T6-L2 injury group, and 20 ablebodied volunteers $(8 \mathrm{M}, 12 \mathrm{~F})$ in the control group. All groups were similar as regards age $(p=0.694)$ and body mass index $(p=0.139)$. Time after injury was similar among the patients with SCI $(p=0.480)$.

Comparisons regarding pulmonary function tests were significantly different among the four groups (Table 2). For all the measurements (except for FEV1/FVC); able-bodied volunteers had better results than patients with SCI; and among the SCI groups, the higher the injury level was, the worse the results for the pulmonary function tests seemed.

Table 3 shows the measurements of diaphragmatic muscle thickness. The diaphragms of $\mathrm{C} 2-\mathrm{C} 4$ group were found to be thicker than those of the controls at endinspirium on the right side $(p=0.035)$. The thickness ratios of C2-C4 group were lower than those of able-bodied volunteers on the right $(p=0.005)$ and left $(p=0.003)$ sides. Right and left side diaphragm measurements were similar within the all SCI groups and able-bodied volunteers (all $p$-values $>0.05$ ).

\section{Discussion}

This study is the first to investigate the association between pulmonary function tests and sonographic diaphragm measurements in different groups of patients with SCI. Our results showed that although the diaphragm muscle thickness at end-inspirium on the right side was thicker and that the thickness ratios on both sides were lower in the $\mathrm{C} 2-\mathrm{C} 4$ injury group, those patients had worse pulmonary function than able-bodied volunteers.

Regulation of the respiratory system is multifactorial. It is well known that the extent of respiratory impairment is related to the injury level, completeness of injury and time after injury [18]. For sure, higher neurological levels result in more paralyzed respiratory muscles and expectedly, paresis/paralysis of some inspiratory muscles (i.e., diaphragm, sternocleidomastoid (SCM), inspiratory intercostal and scalene muscles) may worsen the respiratory failure. If the injury level is below the phrenic motor neurons, the diaphragm and SCM muscles may function normally. Due to incomplete phrenic nerve paresis of our patients in the 
Table 2 Spirometric values of the participants
Table 3 Ultrasonographic diaphragm thickness measurements $($ mean $\pm \mathrm{SD})$

\begin{tabular}{lccccr}
\hline & Able-bodied $(N=20)$ & C2-C4 $(N=7)$ & C5-T5 $(N=14)$ & T6-L2 $(N=21)$ & $P$ \\
\hline FVC, L & $4.4 \pm 0.9$ & $1.9 \pm 0.5$ & $2.9 \pm 0.6$ & $3.9 \pm 1.0$ & $<\mathbf{0 . 0 0 1}$ \\
FVC \% & $108.9 \pm 12$ & $42.7 \pm 6.0$ & $64.6 \pm 14.6$ & $86.8 \pm 15.3$ & $<\mathbf{0 . 0 0 1}$ \\
FEV1, L & $3.7 \pm 0.8$ & $1.4 \pm 0.7$ & $2.6 \pm 0.6$ & $3.4 \pm 0.8$ & $<\mathbf{0 . 0 0 1}$ \\
FEV1 \% & $106.6 \pm 12.8$ & $36.8 \pm 17.3$ & $69.7 \pm 13.9$ & $90.7 \pm 14.3$ & $<\mathbf{0 . 0 0 1}$ \\
FEV1/ FVC, \% & $84.1 \pm 4.6$ & $76.1 \pm 34.8$ & $91.1 .2 \pm 6.7$ & $88.4 \pm 7.9$ & 0.064 \\
FEF 25-75, L/s & $4.2 \pm 1.18^{\mathrm{a}}$ & $2.3 \pm 0.8$ & $3.5 \pm 1.3$ & $4.2 \pm 1.4$ & $\mathbf{0 . 0 0 3}$ \\
FEF 25-75 \% & $98.7 \pm 20.3$ & $52.1 \pm 18.8$ & $77.6 \pm 25.4$ & $93.8 \pm 27.2$ & $<\mathbf{0 . 0 0 1}$ \\
PEF, L/s & $8.1 \pm 1.7$ & $3.3 \pm 1.1$ & $5.6 \pm 1.5$ & $7.0 \pm 2.2$ & $<\mathbf{0 . 0 0 1}$ \\
PEF \% & $99.8 \pm 15.2$ & $38.7 \pm 10.7$ & $62.6 \pm 15.7$ & $76.4 \pm 26.3$ & $<\mathbf{0 . 0 0 1}$ \\
MVV, L/min & $130.0 \pm 35.4$ & $48.2 \pm 21.5$ & $100.4 \pm 22.9$ & $109.3 \pm 33.9$ & $<\mathbf{0 . 0 0 1}$ \\
VC, L & $4.3 \pm 1.0$ & $1.6 \pm 0.6$ & $2.9 \pm 0.6$ & $3.8 \pm 1.0$ & $<\mathbf{0 . 0 0 1}$ \\
\hline
\end{tabular}

Values are given as mean $\pm \mathrm{SD}$

$\%$ values are percent predicted values for lung function

$F V C$ forced vital capacity, FEV1 forced expiratory volume in one second, FEV1/FVC ratio of forced expiratory volume in $1 \mathrm{~s}$ to forced vital capacity, $F E F \quad 25-75$ forced expiratory flow rate, $P E F$ peak expiratory flow rate, $M V V$ maximum voluntary ventilation, $V C$ vital capacity

${ }^{a}$ Bold $P$-values indicate statistical significance

\begin{tabular}{llllll}
\hline Thickness (mm) & Able-bodied $N=20$ & C2-C4 N=7 & C5-T5 $N=14$ & T6-L2 N=21 & $P$ \\
\hline R - end inspirium & $2.0 \pm 0.5^{\mathrm{a}}$ & $2.7 \pm 0.7^{\mathrm{a}}$ & $2.2 \pm 0.4$ & $2.1 \pm 0.5$ & $\mathbf{0 . 0 3 5}$ \\
R - end expirium & $1.9 \pm 0.4$ & $2.2 \pm 0.4$ & $2.0 \pm 0.4$ & $2.0 \pm 0.4$ & 0.272 \\
R - max & $4.5 \pm 0.9$ & $4.1 \pm 1.2$ & $4.5 \pm 1.1$ & $3.9 \pm 1.3$ & 0.330 \\
R - ratio & $1.5 \pm 0.5^{\mathrm{a}}$ & $0.8 \pm 0.4^{\mathrm{a}}$ & $1.3 \pm 0.5$ & $1.0 \pm 0.4$ & $\mathbf{0 . 0 0 5}$ \\
L - end inspirium & $2.0 \pm 0.4$ & $2.2 \pm 0.6$ & $2.0 \pm 0.4$ & $2.2 \pm 0.4$ & 0.600 \\
L - end expirium & $1.8 \pm 0.3$ & $1.9 \pm 0.4$ & $1.9 \pm 0.4$ & $1.9 \pm 0.4$ & 0.684 \\
L - max & $4.5 \pm 1.2$ & $3.4 \pm 1.0$ & $4.5 \pm 0.8$ & $3.9 \pm 1.2$ & 0.051 \\
L - ratio & $1.6 \pm 0.7^{\mathrm{a}}$ & $0.8 \pm 0.5^{\mathrm{a}}$ & $1.4 \pm 0.6$ & $1.0 \pm 0.4$ & $\mathbf{0 . 0 0 3}$ \\
\hline
\end{tabular}

max maximum inspiration, $R$ right, $L$ left

ratio; [thickness max-inspiration - thickness end-expiration] $/$ thickness end-expiration

andicate statistical significance between the $\mathrm{C} 2-\mathrm{C} 4$ level injury group and controls.

Bold $P$-values indicate statistical significance
C2-C4 injury group (six of them had only one affected (C5) motor nerve injury); C3 and C4 motor nerves seemed to have compensated during the inspiration work. Although the contractile capacity of the diaphragm was decreased in the $\mathrm{C} 2-\mathrm{C} 4$ injury group, the diaphragm might have performed its highest contraction capacity during inspiration, as it is the sole inspiratory muscle with SCM. Further, the possible increase of inspiration frequency might have led to hypertrophy of the diaphragm muscle as well [19].

Although there are not many studies in the pertinent literature, West et al. [16] showed significant increase in the diaphragm thickness but without significant effects on the lung function (pulmonary function tests) after six weeks of pressure threshold inspiratory muscle training in cervical level SCI rugby players. In their study, although the diaphragm showed hypertrophy, its thickening did not make a change on the pulmonary function tests. In our study, although spirometric measurements were significantly different among the groups, thickening ratios were significantly different only between patients with tetraplegia and able-bodied participants. Additionally, (end-expiration and end-inspiration) thickness measurements of the diaphragm was higher in patients with tetraplegia. This may actually be another interesting topic for future research, in the sense that if the injury level is lower than the cervical level, hypertrophy of the diaphragm might also have a positive effect on the pulmonary function tests.

In another study, Hardy et al. [14] studied three patients with cervical SCI. They measured the magnitude and direction of the diaphragm movement during tidal and maximal inspiratory breathing using B-mode sonography. During the measurements, the diaphragm moved in a caudal 
direction in two participants and in a cephalad direction in one participant. They could not fully explain the difference of diaphragm movements with the level of injury.

Our study had some limitations. First, lung volumes were measured in the sitting position, and US measurements were performed in the supine position for all participants. Although the diaphragm thickness can be measured without gravity in the supine position, diaphragm measurements and lung volumes could actually be affected. Furthermore, considering the fact that the level and severity of injury is known to affect pulmonary function tests in patients with SCI, the distribution of neurological levels might have also affected our study results. The small sample size and participant diversity might explain the failure to demonstrate some significant differences among the subgroups. Likewise, the number of patients in different groups were small to assess any kind of correlation between sonographic and spirometric parameters. Lastly, gender and smoking habits were different among the groups which might have clouded the results as well.

\section{Conclusion}

We found that although patients with high-level SCI had worse pulmonary function and decreased contractile capacity of the diaphragm, they had thicker diaphragm muscles than controls. This might have been due to the compensatory effect of the diaphragm (performing its maximum contraction capacity and increasing frequency of inspiration) against its partial/total denervation and denervation of other respiratory muscles, respectively. Further studies with larger sample sizes are required for evaluating the relationship between muscle morphology and pulmonary function tests.

\section{Data archiving}

The datasets generated and/or analysed during the current study are available from the corresponding author on reasonable request

Author contributions FUM, AMU, GTO were responsible for designing protocol. HE, MA were responsible for collecting data. MK was responsible for statistical analysis. FK and LO provided feedback on the manuscript.

\section{Compliance with ethical standards}

Conflict of interest The authors declare that they have no conflict of interest.

Ethical statement The presidency of clinical research ethics committee approved the study with the reference number 978 . We certify that all applicable institutional regulations concerning the ethical use of human volunteers were followed during the course of this research.
Publisher's note: Springer Nature remains neutral with regard to jurisdictional claims in published maps and institutional affiliations.

\section{References}

1. Anke A, Aksnes AK, Stanghelle JK, Hjeltnes N. Lung volumes in tetraplegie patients according to cervical spinal cord injury level. Scand J Rehabil Med. 1993;25:73-7.

2. Radulovic M, Schilero GJ, Weeht JM. Airflow obstruction and reversibility in spinal cord injury: evidence for functional sympathetic innervation. Arch Phys Med Rehabil. 2008;89: 2349-53.

3. Mateus SR, Beraldo PS, Horan TA. Maximal static mouth respiratory pressure in spinal cord injured patients: correlation with motor level. Spinal Cord. 2007;45:569-75.

4. McCool FD, Tzelepis GE. Dysfunction of the diaphragm. N Engl J Med. 2012;366:932-42.

5. Summerhill EM, El-Sameed YA, Glidden TJ, McCool FD. Monitoring recovery from diaphragm paralysis with ultrasound. Chest. 2008;133:737-43.

6. Ueki J, De Bruin PF, Pride NB. In vivo assessment of diaphragm contraction by ultrasound in normal subjects. Thorax. 1995;50:1157-61.

7. McCool FD, Conomos P, Benditt JO, Cohn D, Sherman CB, Hoppin FG Jr. Maximal inspiratory pressures and dimensions of the diaphragm. Am J Respir Crit Care Med. 1997;155: 1329-34.

8. Wait JL, Nahormek PA, Yost WT, Rochester DP. Diaphragmatic thickness-lung volume relationship in vivo. J Appl Physiol. 1989;67:1560-8

9. Cohn D, Benditt JO, Eveloff S, McCool FD. Diaphragm thickness during inspiration. J Appl Physiol. 1997;83:291-6.

10. Houston JG, Angus RM, Cowan MD, McMillan NC, Thomson NC. Ultrasound assessment of normal hemidiaphragmatic movement: relation to inspiratory volume. Thorax. 1994;49:500-3.

11. Yamaguti WPS, Paulin E, Shibao S, Kodaira S, Chammas MC, Carvalho CRF. Ultrasound evaluation of diaphragmatic mobility in different postures in healthy subjects. $\mathrm{J}$ Bras Pneumol. 2007;33:407-13.

12. Almeida IC, Clementino AC, Rocha EH, Brandao DC, Dornelas, de Andrade A. Effects of hemiplegy on pulmonary function and diaphragmatic dome displacement. Respir Physiol Neurobiol. 2011;188:196-201.

13. Kim M, Lee K, Cho J, Lee W. Diaphragm thickness and inspiratory muscle functions in chronic stroke patients. Med Sci Monit. 2017;23:1247-53.

14. Hardy F, Walker J, Sawyer T. Sonographic measurement of diaphragm movement in patients with tetraplegia. Spinal Cord. 2009;47:832-4.

15. West CR, Campbell IG, Shave RE, Romer LM. Resting cardiopulmonary function in Paralympic athletes with cervical spinal cord injury. Med Sci Sports Exerc. 2012;44:323-9.

16. West CR, Taylor BJ, Campbell IG, Romer LM. Effects of inspiratory muscle training on exercise responses in Paralympic athletes with cervical spinal cord injury. Scand J Med Sci Sports. 2014;24:764-72.

17. Benditt JO. Pathophysiology of neuromuscular respiratory diseases. Clin Chest Med. 2018;39:297-308.

18. Schilero GJ, Spungen AM, Bauman WA, Radulovic M, Lesser M. Pulmonary function and spinal cord injury. Respir Physiol Neurobiol. 2009;166:129-41.

19. Hoh DJ, Mercier LM, Hussey SP, Lane MA. Respiration following spinal cord injury: evidence for human neuroplasticity. Respir Physiol Neurobiol. 2013;189:450-64. 